\title{
Chronic Lymphocytic Leukemia with Divergent Richter's Transformation into a Clonally Related Classical Hodgkin's and Plasmablastic Lymphoma: A Case Report
}

\author{
Gorana Gasljevic $^{a} \quad$ Mateja Grat ${ }^{b}$ Veronika Kloboves Prevodnik ${ }^{c}$ \\ Biljana Grcar Kuzmanov ${ }^{a}$ Barbara Gazic ${ }^{a}$ Luca Lovrecic $^{d}$ Helena Podgornik ${ }^{e}$ \\ ${ }^{a}$ Department of Pathology, Institute of Oncology, Ljubljana, Slovenia; ${ }^{b}$ Department of \\ Hematology, General Hospital Celje, Celje, Slovenia; 'Department of Cytology, Institute of \\ Oncology, Ljubljana, Slovenia; ${ }^{d}$ Clinical Institute of Medical Genetics, University Medical Center \\ Ljubljana, Ljubljana, Slovenia; 'eDepartment of Hematology, University Medical Center Ljubljana, \\ Ljubljana, Slovenia
}

\section{Keywords}

Chronic lymphocytic leukemia · Richter's transformation · Classical Hodgkin's lymphoma ·

Plasmablastic lymphoma $\cdot$ Trisomy 12

\section{Abstract}

Chronic lymphocytic leukemia (CLL) typically pursues a prolonged course. Its transformation into a more aggressive lymphoma occurs in $2-8 \%$ of all patients. Most commonly, diffuse large B-cell lymphoma develops. Transformation into a classical Hodgkin's lymphoma (CHL) occurs in $<1 \%$. Plasmablastic transformation has been only rarely reported. Cases of synchronous divergent transformation of CLL into a composite lymphoma are exceedingly rare. We describe the unique occurrence of the transformation of a long-standing CLL into a synchronous clonally related $\mathrm{CHL}$ as well as plasmablastic lymphoma (PBL) in an 85 -year-old female patient. After 10 years of asymptomatic $C L L$, our patient was treated with a rituximab-chlorambucil scheme in combination with pegfilgrastim for recurrent infections and the development of $B$ symptoms. Five cycles (of six planned) were administrated with no adverse effects. After the fifth cycle, lymphadenopathy with pronounced B symptoms appeared. Histology showed the presence of $\mathrm{CHL}$ in the lymph node, while the bone marrow was infiltrated by PBL. Our patient died in sepsis not receiving further specific oncologic treatment due to her poor general condition. Additional cytogenetic and molecular studies showed that this was a case of mutated CLL with trisomies of chromosomes 12,3 , and 18 (a rare specific +12 plus other-non +19 CLL subgroup). The presence of trisomy 12 has also been proved in plasmablasts and in $\mathrm{CHL}$ cells. 
Gasljevic et al.: Divergent Transformation of Chronic Lymphocytic Leukemia

\section{Introduction}

Chronic lymphocytic leukemia (CLL) is an indolent B-cell lymphoproliferative neoplasm representing the most common leukemia of adults in the Western world [1]. It typically pursues a prolonged course. Its transformation into a more aggressive lymphoma is termed Richter's syndrome (RS) and occurs in 2-8\% of all CLL patients [1]. Most commonly, diffuse large B-cell lymphoma (DLBCL) develops [2] that can be either clonally related (80\%) or unrelated (20\%) [2, 3]. Transformation into a classical Hodgkin's lymphoma (cHL) occurs in $<1 \%[2,3]$, while plasmablastic transformation (PBL-T) has only rarely been reported [4-7]. Synchronous divergent transformation of CLL into a composite lymphoma is exceedingly rare $[6,8-12]$.

Herein, we describe the unique occurrence of the transformation of a long-standing CLL into a synchronous cHL in the lymph node (LN) associated with a PBL-T in the bone marrow (BM).

\section{Case Presentation}

A 74-year-old woman was seen from 2004 until 2008 because of mild leukopenia. In spite of an extensive evaluation, the causes were not been identified. In 2008, neutropenia aggravated. There was no organomegaly. Complete and differential blood count revealed mild leukopenia $\left(2.9 \times 10^{9} / \mathrm{L}\right)$, moderate neutropenia $\left(0.8 \times 10^{9} / \mathrm{L}\right)$, relative lymphocytosis (Ly $58 \%), \mathrm{Hb} 135 \mathrm{~g} / \mathrm{L}$, and normal platelet count ( $\left.\operatorname{Tr} 170 \times 10^{9} / \mathrm{L}\right)$. Peripheral blood and BM smears were slightly hypercellular showing the presence of small lymphocytes with round nucleus, clumped chromatin, inconspicuous nucleoli, and scant cytoplasms, while trephine BM biopsy showed 10\% infiltration with a low-grade B-cell non-Hodgkin's lymphoma, consistent with CLL/SLL. Fluorescence in situ hybridization (FISH) showed a small clone of trisomy 12 (13\% of all cells) in the BM which was of a comparable size (16\%) 2 years later. The following probe sets were applied on previously cultivated BM cells: 11q deletion (ATM), trisomy 12 (GLI), 13q14 deletion (DLEU), 17p deletion (TP53), and t(11;14) (CCND1/IGH). The CLL FISH panel includes Kreatech (Leica Biosystems, Buffalo Grove, USA) ON TP53/ DLEU, ON ATM/GLI, and the Vysis (Abbott Molecular, Des Plaines, USA) LSI IGH/CCND1 Dual Fusion translocation probe. Internal cutoffs for positivity were previously determined as: $5 \%$ for $11 \mathrm{q}$ deletion, $4 \%$ for trisomy $12,4 \%$ for $13 q$ deletion, $6 \%$ for $17 p$ deletion, and $2 \%$ for 11q13/14q32 rearrangement.

The patient was diagnosed with CLL clinical stage Rai 0 . Since she was asymptomatic and her laboratory status was stable, she was observed until the end of 2012, when bicytopenia developed (Leu $3.2 \times 10^{9} / \mathrm{L}$, neutrophils $16 \%$, Hb $120 \mathrm{~g} / \mathrm{L}$, Tr $149 \times 10^{9} / \mathrm{L}$ ). Repeated BM biopsy showed progression of disease with $70-80 \%$ infiltration by CLL (Fig. 1a-d). FISH confirmed only trisomy 12 in $16 \%$ of all cells. Hematologists decided for further observation only. In the second half of 2014, our patient experienced a few episodes of stomatitis, weight loss, and drenching night sweats. On the basis of the clinical picture, bicytopenia, and 70-80\% CLL BM infiltration, immunochemotherapy was introduced according to a rituximab-chlorambucil scheme in combination with pegfilgrastim. Five cycles (of six planned) were administrated with no adverse effects.

After the fifth cycle in May 2015, the patient developed a fever of undulatory type, night sweating, and inguinal-iliac lymphadenopathy with $\mathrm{LN}$ measuring up to $7 \mathrm{~cm}$. Abdominal and thoracic CT showed enlarged mediastinal and para-aortic LN. Histological examination of the excised inguinal LN showed the effacement of the architecture with scattered Reed-Sternberg and Hodgkin (RS\&H) cells in the background of heterogeneous inflammatory cells including 


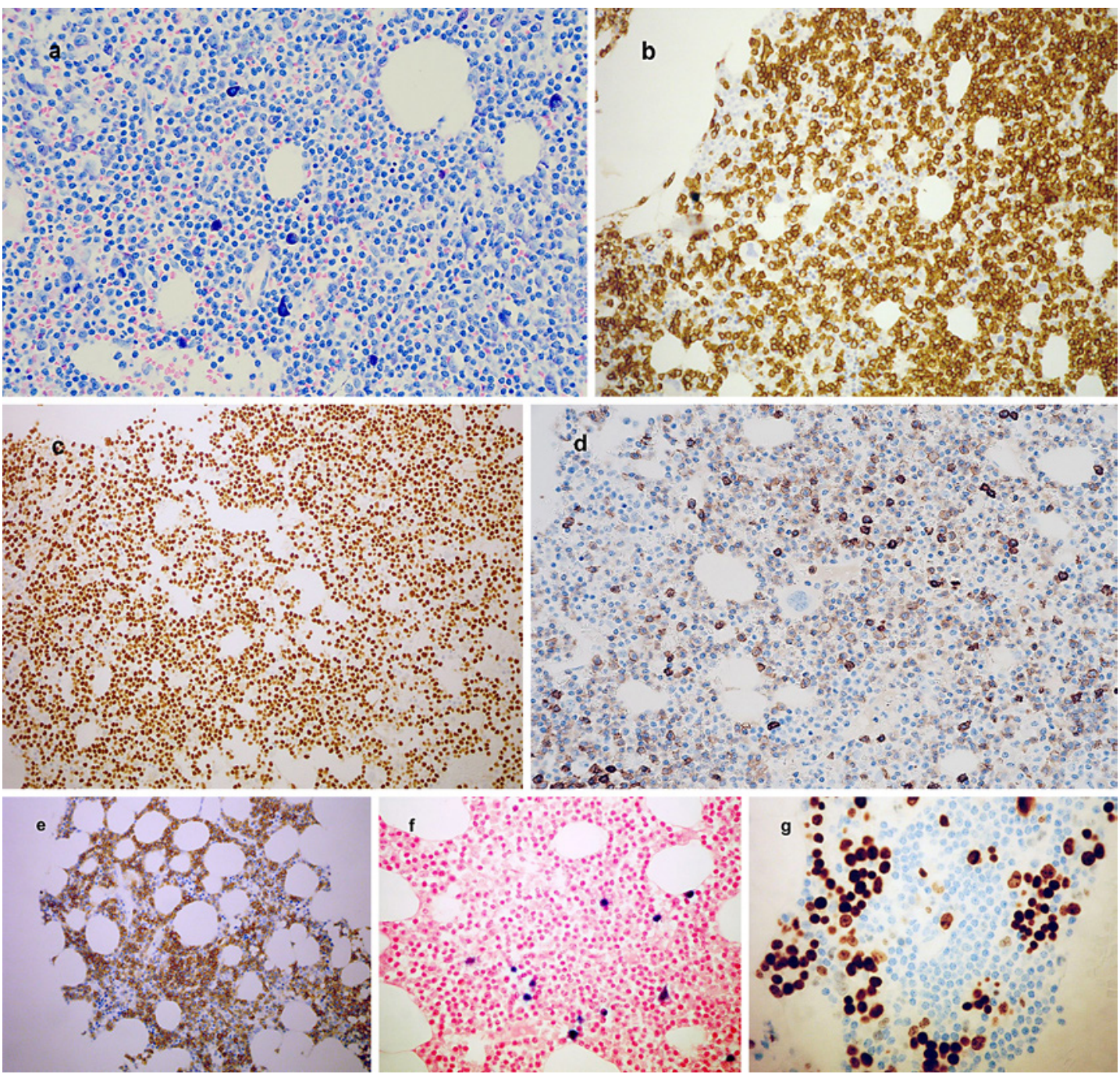

Fig. 1. Bone marrow infiltrated by CLL cells. a Giemsa, $\times 400$. b CD20, $\times 400$. c PAX5, $\times 200$. d CD5, $\times 400$. e CD23, $\times 200$. $\mathbf{f}$ Lambda ISH, $\times 400 . \mathbf{g}$ MIB- $1, \times 400$. The proliferative rate of neoplastic tissue is low, there are some islands of residual orthotopic hematopoiesis showing a higher proliferative rate.

histiocytes, small lymphocytes, plasma cells, and eosinophils (Fig. 2a). Immunohistochemical studies showed positivity of RS\&H cells for CD30 (Fig. 2b), focal positivity for CD15 (Fig. 2c), PAX5 (Fig. 2d), CD79a (Fig. 2e), and MUM1. EBV-LMP and in situ hybridization (ISH) for EBVencoded RNA (EBER) were both negative (Fig. 2g) as well as CD20 (Fig. 2f) and ISH lambda. The morphology and immunophenotypic profile supported the diagnosis of cHL, mixed cellularity.

On the other side, BM biopsy performed for cHL staging showed at least $60 \%$ diffuse interstitial infiltration with large plasmablastic cells (Fig. 3a and b) that were immunohistochemically positive for CD138 (Fig. 3c), weakly, focally for PAX5 (Fig. 3d), bcl2, and EMA with lambda light chain restriction (Fig. 3f). CD20 (Fig. 3e), CD3, CD5, CD10, CD30, bcl6, CD56, EBV-LMP, and EBER (Fig. 3g) were negative. Proliferative fraction of these cells exceeded 90\% (Fig. 3h). Findings in the BM were consistent with a PBL-T. Our patient's condition deteriorated quickly, and she died in sepsis 2-3 weeks after the LN biopsy not receiving any further oncologic treatment because of her poor general condition. 

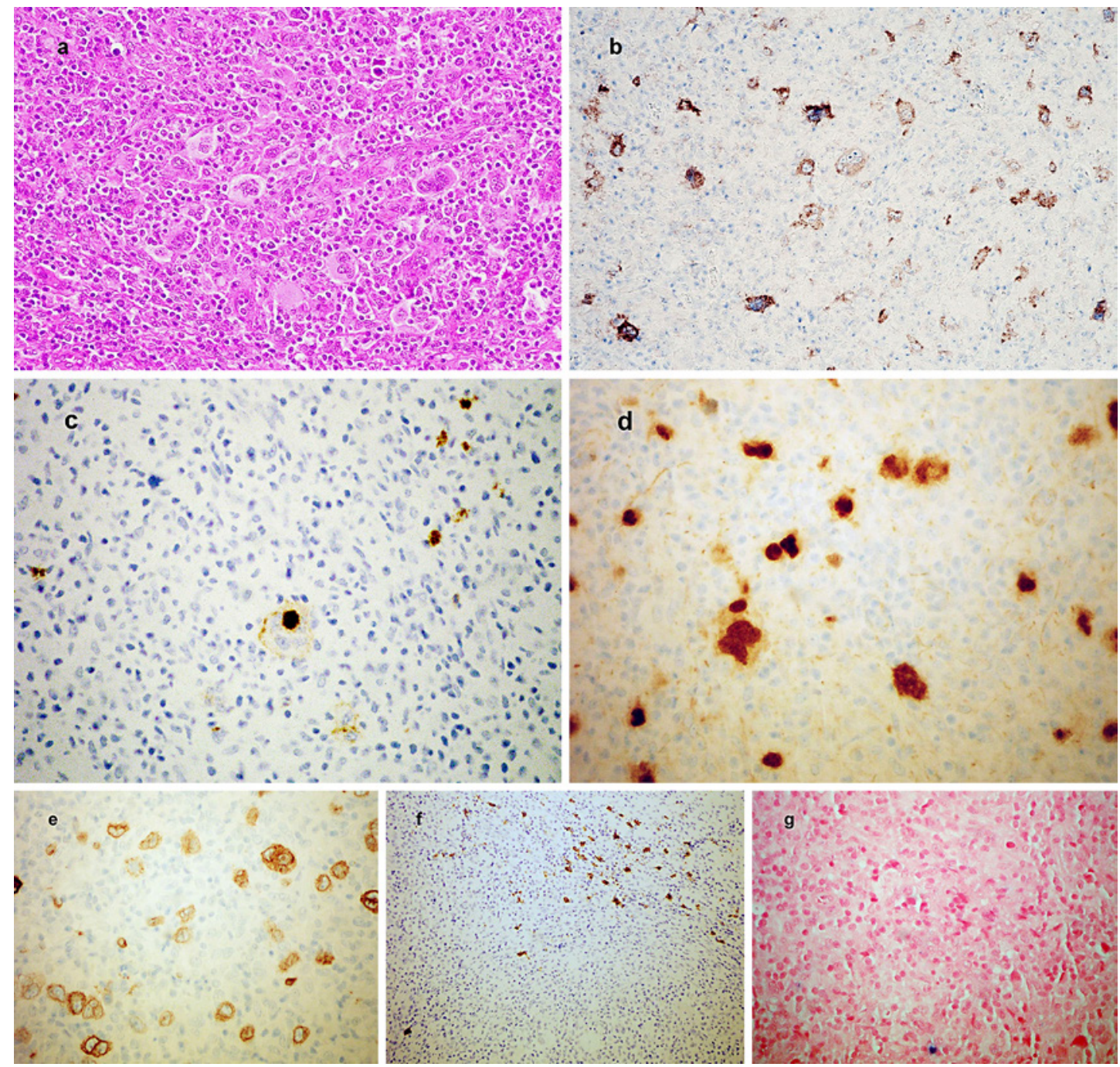

Fig. 2. Classical Hodgkin's lymphoma in a lymph node. a H\&E, $\times 400$. b CD30, ×400. c CD15, ×400. d PAX5, $\times 400$. e CD79a, $\times 400$. f CD20, ×200. g Lambda ISH, $\times 200$. Of notice, there are only a few, residual, CD20/ PAX5/CD79a-positive, small, lymphocytes in the background. Background lymphocytic population consisted mainly of T lymphocytes (not shown).

Additional polymerase chain reaction (PCR)-based immunoglobulin gene rearrangement studies (IgHV) were performed on BM biopsies with CLL from 2013 and PBL-T from 2015 as well as on the inguinal LN. PCR of the CLL showed the presence of rearrangement in the IgHV gene (Fig. 4a). In IgH-C reaction, the same products were present in LN (Fig. 4b) and BM, but no microdissection of RS\&H cells was performed. PCR for IgHV of the BM with PBL-T failed because of hyperfragmented DNA.

Since metaphase chromosomes for conventional chromosome analysis were not available, we performed a comparative genomic hybridization (CGH) analysis to detect possible additional genetic aberrations in the CLL sample from 2012. DNA for the CGH analysis was isolated from CLL cells previously cultivated and harvested for cytogenetic analysis, according to the manufacturer's protocol using the Qiagen kit (Qiagen, Valencia, CA, USA). The quality and concentration parameters of the DNA were measured on a NanoDrop2000c spectrophotometer (Thermo Fisher Scientific Inc.) and a Qubit 2.0 fluorometer (Life Technologies Inc.). 


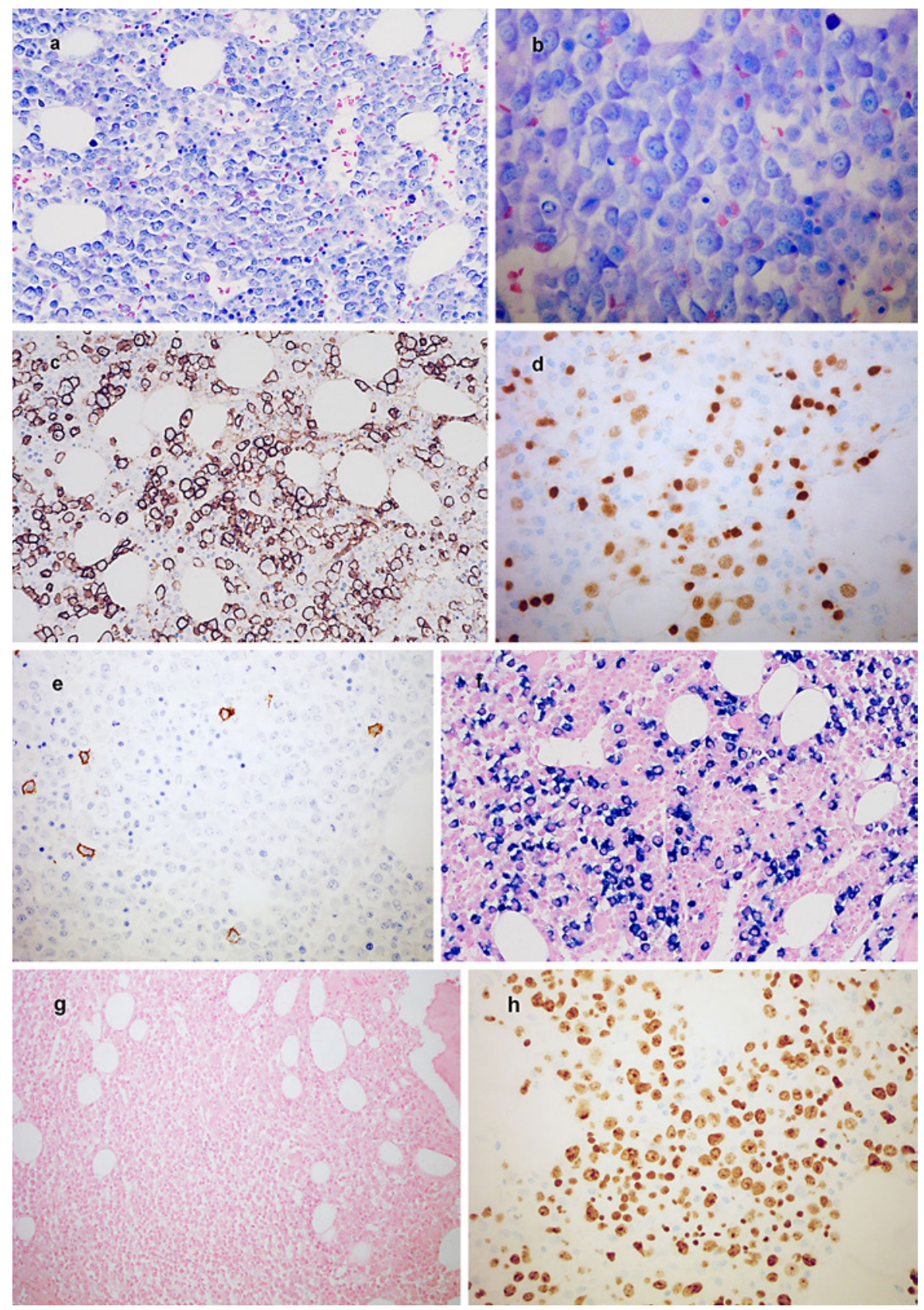

Fig. 3. Bone marrow infiltrated by plasmablasts. a Giemsa, $\times 400$. b Giemsa, $\times 600$. c CD138, $\times 400$. d PAX5, $\times 400$. e CD20, $\times 400$. $\mathbf{f}$ Lambda ISH, $\times 400 . \mathbf{g}$ EBER, $\times 400$. h MIB- $1, \times 400$. The proliferative rate of neoplastic tissue is high, exceeding 90\%. Of notice, CD20 and PAX5 stain only a few, rare, residual, small lymphocytes, while plasmablasts are CD20 negative and focally weakly positive for PAX5.

Following the sample extraction, the DNA was processed according to the Agilent protocol (Version 7.3, March 2014) using commercially available male and female genomic DNA (Agilent Technologies, Human Reference DNA, Male and Female). The Agilent SurePrint G3 Unrestricted CGH ISCA v2, $8 \times 60 \mathrm{~K}$ microarrays were used which provide a practical average resolution of $100 \mathrm{~kb}$. The array images were acquired using the Agilent laser scanner G2565CA. The image files were quantified using the Agilent Feature extraction software for Cytoge- 
Fig. 4. Immunoglobulin clonality profile, sequencing analysis of IgHV-D-J rearrangements in a CLL specimen (a), a cHL specimen (b). of notice, no microdissection of RS-H cells was performed.

Fig. 5. Trisomies 3 (a, d), 12 (b, e), and 18 (c, f) on paraffin embedded sections from lymph nodes $(\mathbf{a}, \mathbf{b}, \mathbf{c})$ and bone marrow (d, e, f) confirmed by FISH using Abbott DNA LSI and CEP probes. Analysis done at the time of transformation.
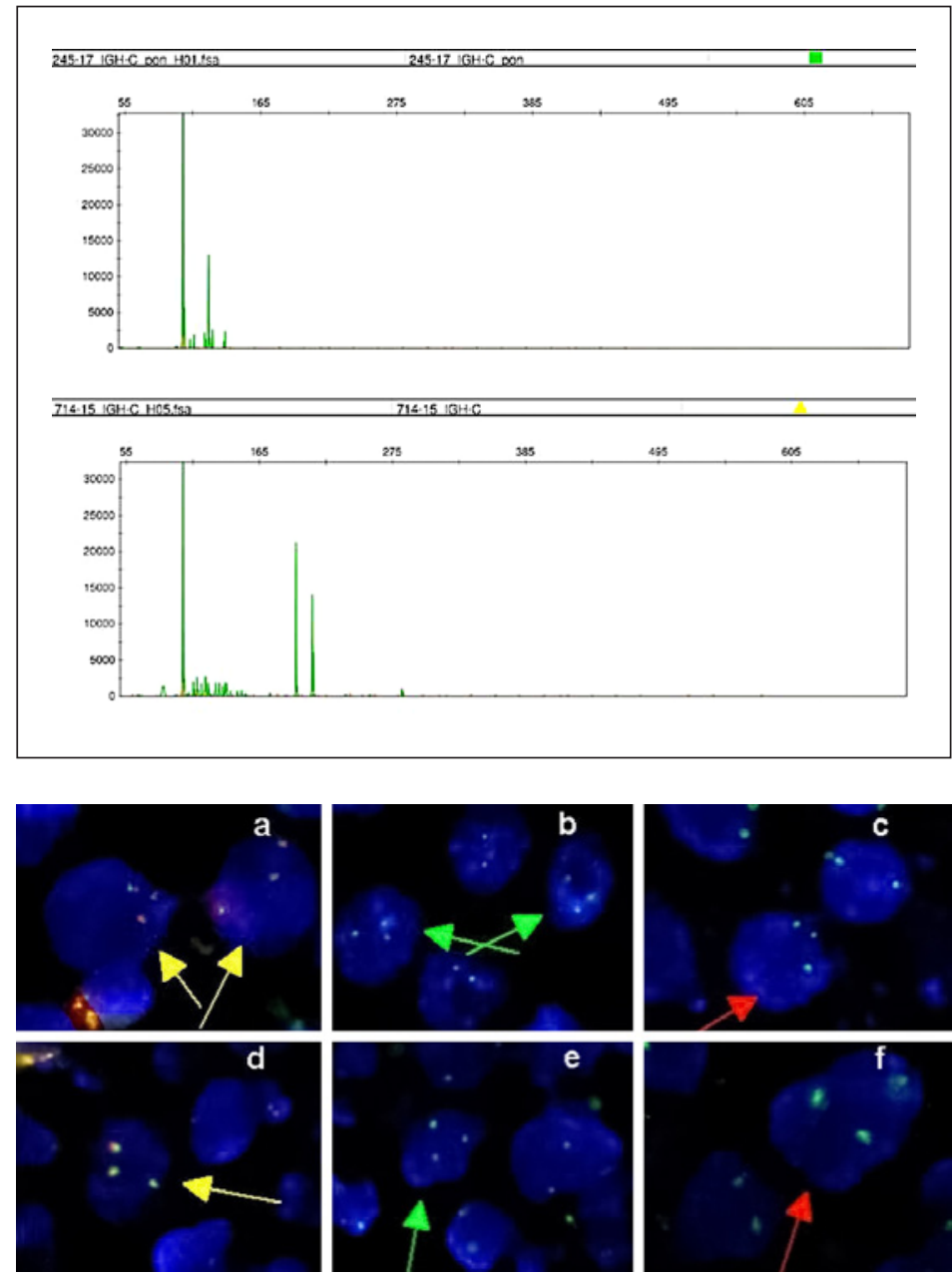

nomics 3.0 and analyzed with the Agilent Cytogenomics 3.0 software (Agilent Technologies). Microarray analysis of the sample from 2012 confirmed mosaic trisomy 12 in approximately $40 \%$ of the sample. In addition, the analysis showed a mosaic trisomy 3 in approximately $43 \%$ and mosaic trisomy 18 in approximately $46 \%$ of the sample. Trisomies 3 and 18 were consequently confirmed by FISH in the BM sample from 2014 in 35 and 19\% of all cells, respectively. For trisomies 12, 3, and 18 detection, we applied LSI BCL6, CEP12, and CEP18 probes (Abbott Molecular, Des Plaines, USA). CGH could not have been performed in the PBL-T cells because of lack of suitable material.

Interphase FISH was finally performed on paraffin-embedded sections of LN and BM infiltrated with PBL-T. FISH analysis on the paraffin sections of LN and BM at the time of transformation confirmed the presence of cells harboring all three trisomies in LN and BM (Fig. 5a-f). Considering the size of the nucleus, the majority of the cells in the LN corresponded to lymphocytes. However, there were also bigger cells with trisomies. To selectively target trisomies on RS\&H cells, the paraffin sections were stained by H\&E first. The cells were captured by a black/white camera for cytogenetic analysis (Fig. 6a). After decolorization, FISH was done on the sections and the same cells were captured again. Although some of them, especially polynuclear cells, had very fragile nuclei and did not stain by DAPI, we could observe three clear signals for trisomy 12 on a few of them (Fig. 6b).

FISH for MYC (8q24) rearrangement (Abbott Molecular, IL) showed no rearrangement of the MYC gene neither in PBL-T nor in RS\&H cells. 
Fig. 6. a Mononuclear ReedSternberg cell in the lymph node. b FISH was done on previously stained sections by Abbott DNA probe CEP12.
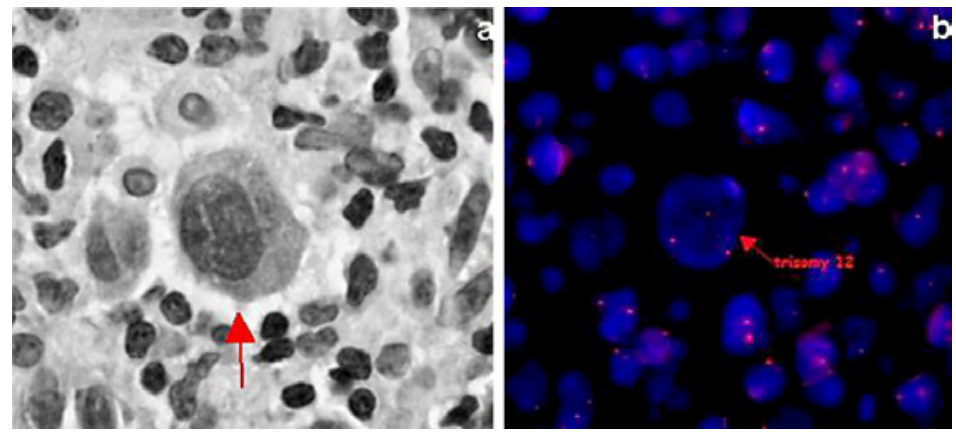

\section{Discussion/Conclusion}

CLL can have a heterogeneous clinical course ranging from an indolent disease that may never require treatment to a rapidly progressive disease that includes histological progression into a high-grade lymphoma; transformation rate is $0.5-1 \%$ per year [1-3]. The majority of transformations are into DLBCL, rarely into a cHL. Other types of transformations are rarer. Risk factors associated with the development of a RS include a combination of clinical features $[1,13]$, biologic characteristics [13], and specific genetic aberrations [2, 3].

CLL transformation into a cHL is a rare event. The vast majorities of cHL cases occur in mutated CLL, are EBV-positive, and are unrelated to CLL clone [2, 3]. In our case, we observed the presence of the same trisomies in CLL cells as well as in RS\&H cells pointing at the high probability that the two lymphomas are clonally related. There are no clearly established risk factors that could identify the subgroup of patients predestined to cHL transformation, but one of them could be immunosuppression associated with the biology of CLL/SLL or prior treatment [1-3]. Clinical outcome of such an occurrence is poor and significantly worse than in patients with de novo cHL [1-3]. The more aggressive course is not related to Ann Arbor stage or the cHL subtype [1-3] but is especially observed when an active CLL continues at the time of transformation, which was also the case in our patient.

PBL-T has only rarely been reported [4-7]. A plasmablastic phenotype is characteristic of plasmablastic lymphoma (PBL), an aggressive disease mainly presenting in extra-nodal sites, often associated with immunodeficiency and EBV infection[1]. In some of the reports $[4,7]$, CLL and PBL-T clones were probably clonally related. Conclusion on that were based on the presence of the same light chain restriction in CLL and PBL-T, but molecular studies on immunoglobulin gene rearrangement have been studied in only one case [4] that showed an unmutated immunoglobulin gene in both tumors. Clonally unrelated cases usually occur in IgHV-mutated CLL [1]. Although our case was a mutated CLL, FISH analysis showed the presence of the same trisomies in the CLL sample and the transformed clones. PCR in BM with PBL-T failed because of hyperfragmented DNA, so targeted FISH was performed. PBL-T and RS\&H cells were stained first to unambiguously locate their nuclei in the tissue. Then, we consecutively confirmed one of trisomies by FISH, namely +12 , pointing at the high probability of clonal relation between malignancies. In the pathogenesis of PBL, deregulation of MYC gene expression is important $[1,4,7]$. EBV-positive PBL more frequently harbors MYC translocation (75\%) and shows poorer overall survival [1]. PBL-T in our case was EBV negative and did not show MYC rearrangement.

Around $2 \%$ of all +12 CLL cases harbor additional trisomies with +19 as the most prevailing additional trisomy [14]. Considering CLL cytogenetics, our patient corresponds to a specific rare +12 plus other-non +19 CLL subgroup with co-existing trisomies 3 and 18 [15]. In the group of non- 19 trisomies, +3 and +18 are, however, the most frequent although rarely 
observed concomitantly [15]. Subgroups of co-existing trisomy cases demonstrate distinctive clinico-biological profiles and characteristics $[14,15]$. Chromosomal banding analysis was not done but on the basis of the FISH and CGH results we can conclude that the three trisomies were the only genetic changes. Namely, it has been shown that patients within this group have exclusively numerical abnormalities [16] and a mutated IGHV gene [15]. Information on the particular IgHV subset of RS\&H and PBL-T cells would be of great value for decisive confirmation of clonal evolution of CLL into other malignancies as was described in some other publications [17]; however, the same trisomies were observed in our case.

Patients with the +12 plus other-non +19 subgroup of CLL have a more indolent course in comparison to isolated +12 ; the same was observed in our patient. The most notable feature of this group of CLL patients is a significantly increased incidence of clinical/laboratory autoimmune manifestations and other malignancies [18]. Autoimmune manifestations in CLL patients are usually of hematological type [18]. Our patient had been suffering from neutropenia a few years before small clones of CLL have been proved to be present in her BM. Another intriguing fact in this group of patients is also an increased incidence of other malignancies, namely, solid cancers [15], while RS has not been documented. The role of abovementioned specific cytogenetic abnormality in the development of synchronous solid cancers and RS does require further investigation in larger cohorts of patients.

Cases of synchronous divergent transformation of CLL into a composite lymphoma are exceedingly rare [6, 8-12]. They include a case of composite prolymphocytic and cHL [8], CLL transformed into a cHL and anaplastic large cell lymphoma [9], composite DLBCL and cHL [10], cHL and anaplastic plasmacytoma [11], and concurrent cHL and PBL-T [6]. In rare cases, clonal relationships have been proved [9], but the majority of them has not been investigated. In two of those, a divergent transformation similar to the one present in our case was described. Hussaini et al. [11] described a long-standing CLL that transformed into a cHL and anaplastic plasmacytoma in the same LN. PBL expresses plasma cell markers but not the markers of mature B cells. Involvement of $\mathrm{LN}$ is found in $<10 \%$ of all cases but is much more frequent in a posttransplant setting, i.e., in an immunosuppression setting (30\%).

$\mathrm{BM}$ involvement may be seen, but the clinical disease distribution is that of a lymphoma rather than a plasma cell myeloma. Plasmocytic differentiation can turn the differential diagnosis toward anaplastic or plasmablastic plasmacytoma, but the overall background of longstanding CLL treated with multiple chemotherapy regiments argues against the diagnosis of plasmacytoma even if the case was EBV negative. It could very well be that the described case also represents the case of a transformation into cHL and PBL-T. Foo et al. [6] described the case of a long -tanding CLL and synchronous transformation into PBL-T and CHL after induction of therapy with fludarabine. Since neoplastic cells of cHL and PBL-T cells were EBV positive, such a finding was categorized as iatrogenic immunodeficiency-associated lymphoproliferative disorder. Of note, our patient had no reported history of fludarabine treatment and was EBV negative. Although immunodeficiency plays a major role in the development of secondary malignancy in transformations in CLL patients, our case should not be considered as immunodeficiency-related since EBV was negative.

A few differential diagnoses could be debated in our case. The first one is an eventual existence of CLL with RS\&H cells in LN. Although an IgHV analysis showed the presence of the same products in the BM with CLL and LN, talking in the favor of some residual CLL clone present in the LN, we think that the clinical picture strongly supports a diagnosis of transformation. Our patient has not had either splenomegaly nor lymphadenopathy in the whole course of years. The development of lymphadenopathy coincided with the clinical deterioration and development of $\mathrm{B}$ symptoms that is highly characteristic for transformation. The second differential diagnosis to consider is a CLL with plasmacytoid differentiation in the last 
Gasljevic et al.: Divergent Transformation of Chronic Lymphocytic Leukemia

BM specimen. This differential diagnosis can easily be abandoned by morphology of PBL-T cells in comparison to CLL cells and their proliferative rate, PBL-T showing a high proliferative activity while it does not exceed a few percent in CLL.

\section{Conclusion}

In conclusion, herein we described the unique case of a long-standing CLLin an 85-year-old patient in the very rare specific +12 plus other-non+19 CLL subgroup with co-existing trisomies 3 and 18 and transformation into a composite lymphoma, namely cHL and PBL-T. Considering the trisomies observed, it is very likely that both of them are clonally related to the CLL clone. To the best of our knowledge, such a case has never been previously described.

\section{Statement of Ethics}

This case study was approved by the Medical Ethics Committee of the Institute of Oncology, Ljubljana, Slovenia. Informed consent for publication is not applicable. The patient died, and she has not had any living relatives.

\section{Disclosure Statement}

The authors declare that they have no competing interests.

\section{Funding Sources}

The publication of this article was financed by the Slovenian Research Agency (ARRS), grant No. P3-0289. The funder had no role in study design, data collection and analysis, decision to publish, or preparation of the manuscript.

\section{Author Contributions}

G.G.: major contributor in writing the manuscript; made substantial contributions to conception and design, or acquisition of data, or analysis and interpretation of data; M.G.: analyzed and interpreted the patient data regarding the hematological disease and treated the patient. V.K.P.: has been involved in drafting the manuscript or revising it critically for important intellectual content. B.G.K., B.G., and L.L.: have been involved in acquisition, analysis, and interpretation of data; H.P.: made substantial contributions to conception and design, or acquisition of data, or analysis and interpretation of data; major contributor in writing the manuscript.

All authors read and approved the final manuscript.

\section{Availability of Data and Material}

Data sharing is not applicable to this article as no datasets were generated or analyzed during the current study. 
Gasljevic et al.: Divergent Transformation of Chronic Lymphocytic Leukemia

\section{References}

1 Campo E, Ghia P, Montserrat E, Harris NL, Müller-Hermelink HK, Stein H, et al. Chronic lymphocytic leukemia/ Small lymphocytic lymphoma. In: WHO classification of tumors of haematopoietic and lymphoid tissues. 4th ed, revised. Lyon: IARC; 2017.

2 Rossi D, Spina V, Deambrogi C, Rasi S, Laurenti L, Stamatopoulos K, et al. The genetics of Richter's syndrome reveals disease heterogeneity and predicts survival after transformation. Blood. 2011;117(12):3391-401.

3 Mao Z, Quintanilla-Martinez L, Raffeld M, Richter M, Krugmann J, Burek C, et al. IgVH mutational status and clonality analysis of Richter's transformation: diffuse large B-cell lymphoma and Hodgkin lymphoma in association with B-cell chronic lymphocytic leukemia (B-CLL) represent two different pathways of evolution. Am J Surg Pathol. 2007;31(10):1605-14.

4 Martinez D, Valera A, Perez NS, Sua Villegas LF, Gonzalez-Farre B, Sole C, et al. Plasmablastic transformation of low-grade B-cell lymphomas. Report on 6 cases. Am J Surg Pathol. 2013;37(2):272-80.

5 Ramalingam P, Nayak-Kapoor A, Reid-Nicholson M, Jones-Crawford J, Ustun C. Plasmablastic lymphoma with small lymphocytic lymphoma: clinico-pathologic features and review of the literature. Leuk Lymphoma. 2008; 49:1999-2002.

6 Foo WC, Huang Q, Sebastian S, Hutchinson CB, Burchette J, Wang E. Concurrent classical Hodgkin lymphoma and plasmablastic lymphoma in a patient with chronic lymphocytic leukemia/small lymphocytic lymphoma treated with fludarabine: a dimorphic presentation of iatrogenic immunodeficiency-associated lymphoproliferative disorder with evidence suggestive of multiclonal transformability of B-cells by Epstein-Barr virus. Hum Pathol. 2010;41:1802-8.

7 Pan Z, Xie Q, Repertinger S, Richendollar BG, Chan WC, Huang Q. Plasmablastic transformation of low grade CD5+ B-cell lymphoproliferative disorder with MYC gene rearrangements. Hum Pathol. 2013;44:2139-48.

8 O’Sullivan MJ, Kaleem Z, Bolger MJ, Swanson P, Zutter M. Composite prolymphocytoid and Hodgkin transformation of chronic lymphocytic leukemia. Arch Pathol Lab Med. 2000;124:907-10.

9 Van den Berg A, Maggio E, Rust R, Kooistra K, Diepstra A, Poppema S. Clonal relation in a case of CLL, ALCL and Hodgkin composite lymphoma. Blood. 2002;100:1425-9.

10 Michelis FV, Kourti G, Skertsou M, Karmiris T, Rontogianni DP, Harhalakis N. Richter transformation of chronic lymphocytic leukemia into composite diffuse large B cell and Hodgkin lymphoma. Leuk Lymphoma. 2012; 53(11):2302-3.

11 Hussaini MO, Klein J, Xiao J, Frater JL, Kreisel F, Hassan A. Richter syndrome: an unusual case of concurrent small lymphocytic lymphoma, Hodgkin lyphoma and anaplastic plasmacytoma. J App Hematol. 2014;5(1): 23-6.

12 O'Sullivan JM, Kaleem Z, Bolger MJ, Swanson PE, Zutter MM. Composite prolymphocytoid and Hodgkin's transformation of chronic lymphocytic leukemia. Arch Pathol Lab Med. 2000;14:907-9.

13 Cramer P, Hallek M. Prognostic factors in chronic lymphocytic leukemia - what do we need to know?. Nat Rev Clin Oncol. 2011;8:38-47.

14 Sellmann L1, Gesk S, Walter C, Ritgen M, Harder L, Martín-Subero JI, et al. Trisomy 19 is associated with trisomy 12 and mutated IGHV genes in B-chronic lymphocytic leukaemia. Br J Haematol. 2007;138(2):217-20.

15 Baliakas P, Puiggros A, Xochelli A, Sutton LA, Nguyen-Khac F, Gardiner A, et al. Additional trisomies amongst patients with chronic lymphocytic leukemia carrying trisomy 12: the accompanying chromosome makes a difference. Haematologica. 2016 Jul;101(7):e299-302.

16 Del Giudice I, Rossi D, Chiaretti S, Marinelli M, Tavolaro S, Gabrielli S, et al. NOTCH1 mutations in +12 chronic lymphocytic leukemia (CLL) confer an unfavorable prognosis, induce a distinctive transcriptional profiling and refine the intermediate prognosis of +12 CLL. Haematologica. 2012;97(3):437-41.

17 Chen W, Jaffe R, Zhang L, Hill C, Block AM, Sait S, et al. Langerhans cell sarcoma arising from chronic lymphocytic lymphoma/small lymphocytic leukemia: lineage analysis and BRAF V600E mutational study. N Am J Med Sci. 2013;5:386-91.

18 Dearden C. Disease-specific complications of chronic lymphocytic leukemia. Hematol Am Soc Hematol Educ Program. 2008;450-6. 